\title{
Hotelling's Duopoly in a Two-Sided Platform Market on the Plane
}

\author{
Vladimir Mazalov $1,2,3,+, \ddagger$ (iD) and Elena Konovalchikova $4, *, \neq(i)$ \\ 1 Institute of Applied Mathematical Research, Karelian Research Center of the Russian Academy of Sciences, \\ 11, Pushkinskaya str., 185910 Petrozavodsk, Russia; vmazalov@krc.karelia.ru \\ 2 School of Mathematics and Statistics, Qingdao University, Qingdao 266071, China \\ 3 Institute of Applied Mathematics of Shandong, Qingdao 266071, China \\ 4 Laboratory of Digital Technologies in Regional Development, Karelian Research Center of the Russian \\ Academy of Sciences, 11, Pushkinskaya str., 185910 Petrozavodsk, Russia \\ * Correspondence: konovalchikova_en@mail.ru \\ $\dagger$ The work was supported by the Shandong Province “Double-Hundred Talent Plan" (No. WST2017009). \\ $\ddagger$ These authors contributed equally to this work.
}

Received: 15 May 2020; Accepted: 25 May 2020; Published: 27 May 2020

\begin{abstract}
Equilibrium in a two-sided market represented by network platforms on the plane and heterogeneous agents is investigated. The advocated approach is based on the duopoly model which implies a continuum of agents of limited size on each side of the market and examines the agents' heterogeneous utility with the Hotelling specification. The exact values were found for the equilibrium in the case of duopoly in a two-sided market with two platforms on the plane. The dependence of the platforms' benefits on network externalities was investigated. The problem of the optimal location of platforms in the market was considered.
\end{abstract}

Keywords: two-sided platform market; pricing; Hotelling's duopoly on the plane; Nash equilibrium; optimal location of platforms

\section{Introduction}

Digital economy has formed a paradigm of accelerated economic development. A central position in it is occupied by network technologies, which have led to the establishment of network markets. Network markets are set up on network platforms, which are a novel business element in the modern economy, providing benefits for both the platform's operator and the community.

The two-sided market involving a platform is a network type of market, where users belonging to two different groups interact. Consumption by any one of the groups generates external effects on the other group. Assuming a network effect exists between the two sides of the market (network externalities), the problem of optimizing the platform's profit can be formulated. Reference [1] suggested a model of a monopoly platform in a two-sided market with the total number of agents on each side equaling one. The agents have different transport costs to access the platform via Hotelling specification. As an illustration, imagine that agents in the model are sellers and customers in an online store, job-seekers and employers in a job centre, men and women in a dance floor, and so forth. Following the Hotelling specification [2], agents compare their costs before taking the decision about visiting the platform. Our study assumes agents to be heterogeneous and demand to be endogenous, and investigates the platform's behavior and revenues under these assumptions.

A duopoly in a two-sided in-plane platform market is considered, equilibrium in such a market and the dependence of platform revenues on network externalities are determined. For the case of identical agents and identical platforms, the conditions were detected enabling services in such 
platforms to exist under competition, and the exact expressions for the platforms' optimal strategies were found.

Also, the social optimum problem was solved and the dependence of the optimal value on the structure of the two-sided market's costs and network externalities was determined.

A number of papers have been published on network platforms with externalities. Since the analysis of monopolistic markets [3-7], studies in this sphere have been focused on the pricing platforms for markets with different structures, including the problem of the social optimum for private platforms and associations. The aspects addressed were the models of private platforms' competition in different scenarios for homogeneous and differentiated products, bottlenecks, exclusive contracts, as well as the effects of various important factors such as tariffs, market price elasticity, transaction size, and buyers and sellers net surplus.

Rochet and Tirole study the pricing strategy of payment-card type of platforms in two-sided markets, considering both the monopoly case and the duopoly case, with different objective functions of the platforms: either to maximize the profit or to maximize the social welfare [8]. Later on, Rochet and Tirole consider this type of platform model that integrates both usage and membership externalities and study obtain new results on the mix of usage and membership charges [8]. Later on, Rochet and Tirole placed the model of a platform of this type into the context of externalities with membership fees [6].

In Reference [1], which is the closest to our study, Armstrong looked into the pricing strategy in a two-sided market for platforms of the shopping-mall type for the monopoly scenario and a duopoly. In the monopoly case, there were no constraints on the size of the market and the demand function depended on exogenous reasons.

Later on, Armstrong and Wright explored the duopoly competition between two platforms in a linear market, included the Hotelling specification in the model, and considered three cases: (1) product differentiation on both sides; (2) products differentiation on one side only; (3) no product differentiation on either side [9]. For models (1) and (2), the agents' utilities were large enough for using the services of at least one of the platforms. For model (3), the agents were assumed to be homogeneous, and only the network effect was taken into account.

Where in References $[1,9,10]$ each agent is supposed to choose one of the platforms for the service, agents in the model from Reference [11] are not allowed to join any platform if they do not benefit from it. It was demonstrated in the latter paper that assuming a limited market size, heterogeneous agents and endogenous demand, the optimal strategy always results in a corner solution, whether the goal is to maximize the platform's profit or social welfare. Also, the conditions under which the monopoly is socially optimal were found.

Another important issue associated with platform economy is the optimal platform location. This problem was investigated for a one-sided market by Hotelling in Reference [2], and then in some models for a linear market [12-14], and in Reference [15] for a market on the plane. Research on the optimal location of platforms is now in an early stage.

Some papers on two-sided platforms investigated pricing in a market depending on the seller's behavior for special platforms [16-21]. Reference [16] dealt with sequential competition between sellers in a two-sided software market, while Reference [20] focused on competition between virtual operators (sellers) in a two-sided telecommunication market.

Research on these issues is a new area in platform economy. In this study we analyze the performance of platforms under the new assumption that the market is in the plane space. This approach results in new intriguing effects, the market boundary is a second-order curve, which breaks the linearity of solutions and, hence, alters the form of the conditions for the feasibility of competitive service and the form of the solutions as such. Furthermore, the problem of platform location in the market is also meaningful. This is demonstrated below for the case of two platforms.

The article is structured as follows-Section 2 describes the model and its distinctions from previous models; Section 3 finds the equilibrium in the model with identical platforms and identical 
agents, while Section 4 finds the solution for the model with identical groups of agents and platforms occupying different positions in the market; Section 5 investigates the optimal location of platforms; Section 6 finds the equilibrium in the general case of the pricing problem; the article is completed with the conclusions and visions for further research in the area.

\section{Description of the Model}

Suppose there are two non-intersecting groups of agents in the market: group 1 (also known as group of buyers), and group 2 (also known as group of sellers). Agents in both groups, whose size equals a unit, are distributed uniformly over a plane in the square $S=[-1,1] \times[-1,1]$. The location of the members of groups 1 and 2 in the square $S$ is defined by the points $\left(x_{1}, y_{1}\right)$ and $\left(x_{2}, y_{2}\right)$ respectively, where $-1 \leq x_{i} \leq 1$ and $-1 \leq y_{i} \leq 1, i=1,2$. Agents from both groups meet in platforms $I$ and $I I$, located at the boundary of the square $S$ in diametrically opposite points, for example, $I(-1,0)$ and $I I(1,0)$.

The following notations are introduced: $n_{i}^{(j)}$ is the size of the group $i$ in the platform $j(i=1,2 ; j=$ $I, I I) ; p_{i}^{(j)}$ is the price of visiting the platform $j$ by an agent of the group $i(i=1,2 ; j=I, I I) ; \alpha, \beta$ are the degrees of the effect that the number of second (first) group agents in the platform has on the payoff of the first (second) group; $t_{i}$ is the effect of the transport costs for visiting the $i$ th platform where $t_{i}>0(i=1,2)$.

In a two-sided market with two platforms, agents in both groups choose between them based on the utility they can derive from visiting the respective platform. For group 1 agents, the utility of visiting the platform $I$, situated in the point $(-1,0)$ has the form

$$
u_{1}^{(I)}=\alpha \cdot n_{2}^{(I)}-p_{1}^{(I)}-\sqrt{\left(x_{1}+1\right)^{2}+y_{1}^{2}} \cdot t_{1}
$$

and the utility of visiting the platform $I I$, in the point $(1,0)$ is

$$
u_{1}^{(I I)}=\alpha \cdot n_{2}^{(I I)}-p_{1}^{(I I)}-\sqrt{\left(x_{1}-1\right)^{2}+y_{1}^{2}} \cdot t_{1} .
$$

In the above expressions, the first term captures the utility from network externality of the other side of the market, the second term is the cost of price the agent has to pay to access the platform, and the third term is essentially the agent's heterogeneous transport cost of accessing the platform, via Hotelling specification.

The boundary between regions of the market for group 1 is found from the equation $u_{1}^{(I)}=u_{1}^{(I I)}$, which is solved to get

$$
\frac{x_{1}^{2}}{s_{1}^{2}}-\frac{y_{1}^{2}}{1-s_{1}^{2}}=1
$$

where $s_{1}=\frac{\alpha-2 \alpha n_{2}^{(I)}+p_{1}^{(I)}-p_{1}^{(I I)}}{2 t_{1}}$, given that $n_{2}^{(I)}+n_{2}^{(I I)}=1$ (see Figure 1). Parameter $s_{1}$ is the coordinate value at which the hyperbola crosses the abscissa axis. In the symmetric case, when the market is divided equally, $s_{1}=0$. If $s_{1}>0\left(s_{1}<0\right)$, then platform I (II) is more attractive for agents.

The market being divided into two regions, the respective numbers of group 1 agents visiting platforms $I$ and $I I$ are

$$
n_{1}^{(I)}=\frac{1}{2}-\frac{1}{2} \int_{0}^{1} s_{1} \sqrt{1+\frac{y^{2}}{1-s_{1}^{2}}} d y
$$




$$
n_{1}^{(I I)}=\frac{1}{2}+\frac{1}{2} \int_{0}^{1} s_{1} \sqrt{1+\frac{y^{2}}{1-s_{1}^{2}}} d y .
$$

The utilities of group 2 agents from visiting platforms I and II are determined similarly, and are equal, respectively

$$
\begin{gathered}
u_{2}^{(I)}=\beta \cdot n_{1}^{(I)}-p_{2}^{(I)}-\sqrt{\left(x_{2}+1\right)^{2}+y_{2}^{2}} \cdot t_{2}, \\
u_{2}^{(I I)}=\beta \cdot n_{1}^{(I I)}-p_{2}^{(I I)}-\sqrt{\left(x_{2}-1\right)^{2}+y_{2}^{2}} \cdot t_{2},
\end{gathered}
$$

and the boundary between market regions for group 2 is

$$
\frac{x_{2}^{2}}{s_{2}^{2}}-\frac{y_{2}^{2}}{1-s_{2}^{2}}=1
$$

where $s_{2}=\frac{\beta-2 \beta n_{1}^{(I)}+p_{2}^{(I)}-p_{2}^{(I I)}}{2 t_{2}}$, given that $n_{1}^{(I)}+n_{1}^{(I I)}=1$. The number of group 2 agents visiting the respective platforms is

$$
\begin{aligned}
& n_{2}^{(I)}=\frac{1}{2}-\frac{1}{2} \int_{0}^{1} s_{2} \sqrt{1+\frac{y^{2}}{1-s_{2}^{2}}} d y, \\
& n_{2}^{(I I)}=\frac{1}{2}+\frac{1}{2} \int_{0}^{1} s_{2} \sqrt{1+\frac{y^{2}}{1-s_{2}^{2}}} d y .
\end{aligned}
$$

We thus arrive at the pricing game for the two platforms $I$ and $I I$, which respectively serve $n_{1}^{(I)}+n_{2}^{(I)}$ and $n_{1}^{(I I)}+n_{2}^{(I I)}$ agents of both groups. The payoffs of platforms I and II in the pricing game are determined as follows:

$$
\begin{gathered}
H^{(I)}\left(p_{1}^{(I)}, p_{2}^{(I)}\right)=n_{1}^{(I)}\left(p_{1}^{(I)}-g_{1}\right)+n_{2}^{(I)}\left(p_{2}^{(I)}-g_{2}\right), \\
H^{(I I)}\left(p_{1}^{(I I)}, p_{2}^{(I I)}\right)=n_{1}^{(I I)}\left(p_{1}^{(I I)}-g_{1}\right)+n_{2}^{(I I)}\left(p_{2}^{(I I)}-g_{2}\right),
\end{gathered}
$$

where $g_{1}$ and $g_{2}$ are the platforms' costs of serving users from the respective groups. Note that service to any group $i=1,2$ in a platform can be provided only if $p_{i}^{(I, I I)} \geq g_{i}$.

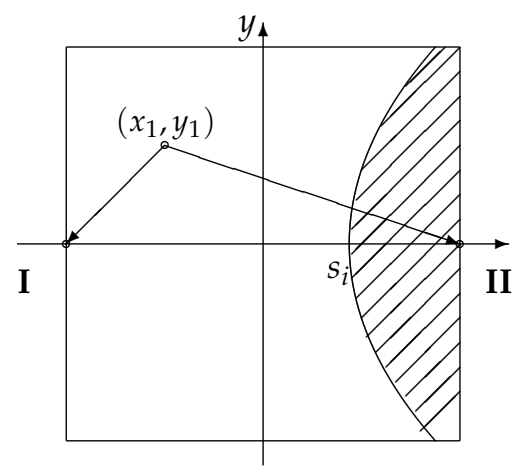

Figure 1. Two-sided platform market on a plane. 


\section{Identical Platforms and Identical Groups of Agents}

Consider the case of identical platforms and identical agents, where the effect of the other group's size on the payoff and the transport costs of agents of the two groups are equal, that is, $\alpha=\beta$ and $t_{1}=t_{2}=t$, and the service costs of the platforms are equal, $g_{1}=g_{2}=g$. In this case, it suffices to find the optimal solution for one of the platforms, for example, for the platform $I$. The price equilibrium is found from the first-order optimality condition $\frac{\partial H^{(I)}}{\partial p_{1}^{(I)}}=0$, which has the following form:

$$
\frac{\partial H^{(I)}}{\partial p_{1}^{(I)}}=\frac{\partial n_{1}^{(I)}}{\partial p_{1}^{(I)}}\left(p_{1}^{(I)}-g\right)+n_{1}^{(I)}+\frac{\partial n_{2}^{(I)}}{\partial p_{1}^{(I)}}\left(p_{2}^{(I)}-g\right)
$$

From Equations (1) and (2) we get that

$$
\begin{gathered}
\frac{\partial n_{1}^{(I)}}{\partial p_{1}^{(I)}}=\frac{\partial n_{1}^{(I)}}{\partial s_{1}} \frac{\partial s_{1}}{\partial p_{1}^{(I)}}=\frac{\partial n_{1}^{(I)}}{\partial s_{1}} \frac{1}{2 t}\left(1-2 \alpha \frac{\partial n_{2}^{(I)}}{\partial p_{1}^{(I)}}\right) \\
\frac{\partial n_{2}^{(I)}}{\partial p_{1}^{(I)}}=\frac{\partial n_{2}^{(I)}}{\partial s_{2}} \frac{\partial s_{2}}{\partial p_{1}^{(I)}}=\frac{\partial n_{2}^{(I)}}{\partial s_{2}} \frac{1}{2 t}\left(-2 \alpha \frac{\partial n_{1}^{(I)}}{\partial p_{1}^{(I)}}\right),
\end{gathered}
$$

from where we find that

$$
\begin{gathered}
\frac{\partial n_{1}^{(I)}}{\partial p_{1}^{(I)}}=\frac{1}{2 t} \frac{\partial n_{1}^{(I)}}{\partial s_{1}}\left(1-\frac{\alpha^{2}}{t^{2}} \frac{\partial n_{1}^{(I)}}{\partial s_{1}} \frac{\partial n_{2}^{(I)}}{\partial s_{2}}\right)^{-1}, \\
\frac{\partial n_{2}^{(I)}}{\partial p_{1}^{(I)}}=-\frac{\alpha}{2 t^{2}} \frac{\partial n_{1}^{(I)}}{\partial s_{1}} \frac{\partial n_{2}^{(I)}}{\partial s_{2}}\left(1-\frac{\alpha^{2}}{t^{2}} \frac{\partial n_{1}^{(I)}}{\partial s_{1}} \frac{\partial n_{2}^{(I)}}{\partial s_{2}}\right)^{-1} .
\end{gathered}
$$

It follows from the symmetry of the problems that in the equilibrium the prices set by both platforms for agents of groups 1 and 2 have to be equal, that is, $p_{1}^{(I)}=p_{1}^{(I I)}=p_{2}^{(I)}=p_{2}^{(I I)}=p$. Furthermore, the size of both groups in the platforms should be equal, that is, $n_{1}^{(I)}=n_{1}^{(I I)}=\frac{1}{2}$ and $n_{2}^{(I)}=n_{2}^{(I I)}=\frac{1}{2}$. It follows from the equality of the prices and numbers of agents in the platforms that $s_{1}=s_{2}=0$ and that

$$
\frac{\partial n_{1}^{(I)}}{\partial s_{1}}=\frac{\partial n_{2}^{(I)}}{\partial s_{2}}=-\frac{1}{2} \int_{0}^{1} \sqrt{1+y^{2}} d y
$$

Denote for brevity $I=\int_{0}^{1} \sqrt{1+y^{2}} d y \approx 1.147$.

Thus, Equations (6) and (7) in the form

$$
\begin{gathered}
\frac{\partial n_{1}^{(I)}}{\partial p_{1}^{(I)}}=-\frac{1}{4 t} I\left(1-\frac{\alpha^{2}}{4 t^{2}} I^{2}\right)^{-1}, \\
\frac{\partial n_{2}^{(I)}}{\partial p_{1}^{(I)}}=-\frac{\alpha}{8 t^{2}} I^{2}\left(1-\frac{\alpha^{2}}{4 t^{2}} I^{2}\right)^{-1},
\end{gathered}
$$


are substituted into (5) to find

$$
\frac{\partial H^{(I)}}{\partial p_{1}^{(I)}}=\left(\frac{\partial n_{1}^{(I)}}{\partial p_{1}^{(I)}}+\frac{\partial n_{2}^{(I)}}{\partial p_{1}^{(I)}}\right)(p-g)+\frac{1}{2}=-\frac{1}{4 t} I\left(1-\frac{\alpha^{2}}{4 t^{2}} I^{2}\right)^{-1}\left(1+\frac{\alpha I}{2 t}\right)(p-g)+\frac{1}{2}=0 .
$$

Derive from here the price in the equilibrium, which is equal to

$$
p=g+\frac{4 t^{2}\left(1-\frac{\alpha^{2}}{4 t^{2}} I^{2}\right)}{I(2 t+\alpha I)}=g+\frac{2 t}{I}-\alpha=g+\frac{2 t}{\int_{0}^{1} \sqrt{1+y^{2}} d y}-\alpha .
$$

Observe that competition exists only if $p \geq g$, or if

$$
\frac{\alpha}{t} \leq \frac{2}{I} \approx 1.742 \text {. }
$$

Test the sufficient conditions for the maximum of the function $H^{I}\left(p_{1}^{I}, p_{2}^{I I}\right)$. To this end, find the following expressions $A=\frac{\partial^{2} H^{(I)}}{\partial^{2} p_{1}^{(I)}}, B=\frac{\partial^{2} H^{(I)}}{\partial p_{1}^{(I)} \partial p_{2}^{(I)}}$ and $C=\frac{\partial^{2} H^{(I)}}{\partial^{2} p_{2}^{(I)}}$. Considering the symmetry of the problem we have

$$
\begin{gathered}
A=\frac{\partial^{2} H^{(I)}}{\partial^{2} p_{1}^{(I)}}=\frac{\partial^{2} n_{1}^{(I)}}{\partial^{2} p_{1}^{(I)}}\left(p_{1}^{(I)}-g_{1}\right)+\frac{\partial^{2} n_{2}^{(I)}}{\partial^{2} p_{1}^{(I)}}\left(p_{2}^{(I)}-g_{2}\right)+2 \frac{\partial n_{1}^{(I)}}{\partial p_{1}^{(I)}}=\left(\frac{\partial^{2} n_{1}^{(I)}}{\partial^{2} p_{1}^{(I)}}+\frac{\partial^{2} n_{2}^{(I)}}{\partial^{2} p_{1}^{(I)}}\right)(p-g)+2 \frac{\partial n_{1}^{(I)}}{\partial p_{1}^{(I)}} \\
B=\frac{\partial^{2} H^{(I)}}{\partial p_{1}^{(I)} \partial p_{2}^{(I)}}=\frac{\partial^{2} n_{1}^{(I)}}{\partial p_{1}^{(I)} \partial p_{2}^{(I)}}\left(p_{1}^{(I)}-g_{1}\right)+\frac{\partial^{2} n_{2}^{(I)}}{\partial p_{1}^{(I)} \partial p_{2}^{(I)}}\left(p_{2}^{(I)}-g_{2}\right)+\frac{\partial n_{1}^{(I)}}{\partial p_{2}^{(I)}}+\frac{\partial n_{2}^{(I)}}{\partial p_{1}^{(I)}}= \\
=\left(\frac{\partial^{2} n_{1}^{(I)}}{\partial p_{1}^{(I)} \partial p_{2}^{(I)}}+\frac{\partial^{2} n_{2}^{(I)}}{\partial p_{1}^{(I)} \partial p_{2}^{(I)}}\right)(p-g)+\frac{\partial n_{1}^{(I)}}{\partial p_{2}^{(I)}}+\frac{\partial n_{2}^{(I)}}{\partial p_{1}^{(I)}}, \\
C=\frac{\partial^{2} H^{(I)}}{\partial^{2} p_{2}^{(I)}}=\frac{\partial^{2} n_{1}^{(I)}}{\partial^{2} p_{2}^{(I)}}\left(p_{1}^{(I)}-g_{1}\right)+\frac{\partial^{2} n_{2}^{(I)}}{\partial^{2} p_{2}^{(I)}}\left(p_{2}^{(I)}-g_{2}\right)+2 \frac{\partial n_{2}^{(I)}}{\partial p_{2}^{(I)}}=\left(\frac{\partial^{2} n_{1}^{(I)}}{\partial^{2} p_{2}^{(I)}}+\frac{\partial^{2} n_{2}^{(I)}}{\partial^{2} p_{2}^{(I)}}\right)(p-g)+2 \frac{\partial n_{2}^{(I)}}{\partial p_{2}^{(I)}} .
\end{gathered}
$$

It follows from the symmetry of the problem that $\frac{\partial n_{1}^{(I)}}{\partial p_{2}^{(I)}}=\frac{\partial n_{2}^{(I)}}{\partial p_{1}^{(I)}}$ and $\frac{\partial n_{1}^{(I)}}{\partial p_{1}^{(I)}}=\frac{\partial n_{2}^{(I)}}{\partial p_{2}^{(I)}}$, and also that $\frac{\partial^{2} n_{1}^{(I)}}{\partial p_{1}^{(I)} \partial p_{2}^{(I)}}=\frac{\partial^{2} n_{2}^{(I)}}{\partial p_{1}^{(I)} \partial p_{2}^{(I)}}=0$ and $\frac{\partial^{2} n_{1}^{(I)}}{\partial^{2} p_{1}^{(I)}}=\frac{\partial^{2} n_{1}^{(I)}}{\partial^{2} p_{2}^{(I)}}=\frac{\partial^{2} n_{2}^{(I)}}{\partial^{2} p_{2}^{(I)}}=0$. Therefore

$$
A=C=2 \frac{\partial n_{1}^{(I)}}{\partial p_{1}^{(I)}}=-\frac{1}{2 t} I\left(1-\frac{\alpha^{2}}{4 t^{2}} I^{2}\right)^{-1}, \quad B=2 \frac{\partial n_{1}^{(I)}}{\partial p_{2}^{(I)}}=-\frac{\alpha}{4 t^{2}} I^{2}\left(1-\frac{\alpha^{2}}{4 t^{2}} I^{2}\right)^{-1} .
$$

Since the expression $A C-B^{2}=\frac{I^{2}}{4 t^{2}}\left(1-\frac{\alpha^{2} I^{2}}{4 t^{2}}\right)^{-2}\left[1+\frac{\alpha^{2} I^{2}}{4 t^{2}}\right]>0$ when $\frac{\alpha}{t}<\frac{2}{I}$ and since $A<0$, the function $H^{(I)}\left(p_{1}^{(I)}, p_{2}^{(I I)}\right)$ has a maximum at $(p, p)$, where $p$ is found from Formula (8). of agents.

Hence, the following theorem is valid for the case of identical platforms and identical groups (n) 
Theorem 1. In the Hotelling model for a two-sided platform market on a plane with identical groups of agents, competitive service will take place given that

$$
\frac{\alpha}{t}<\frac{2}{\int_{0}^{1} \sqrt{1+y^{2}} d y}
$$

and the price in the equilibrium in this case will have the form (8).

\section{Identical Platforms and Different Groups of Agents}

Suppose agents in the two groups differ in their parameters. Remark that owing to their symmetric location in the market the platforms are identical. In this case, the price equilibrium is derived from the first-order optimality condition for each group of agents in one of the platforms, for example, platform $I$, which has the form $\frac{\partial H^{(I)}}{\partial p_{i}^{(I)}}=0, i=1,2$.

The equation $\frac{\partial H^{(I)}}{\partial p_{1}^{(I)}}=0$ has the form

$$
\frac{\partial H^{(I)}}{\partial p_{1}^{(I)}}=\frac{\partial n_{1}^{(I)}}{\partial p_{1}^{(I)}}\left(p_{1}^{(I)}-g_{1}\right)+n_{1}^{(I)}+\frac{\partial n_{2}^{(I)}}{\partial p_{1}^{(I)}}\left(p_{2}^{(I)}-g_{2}\right)
$$

From the Equations (1)-(4), we find that

$$
\begin{gathered}
\frac{\partial n_{1}^{(I)}}{\partial p_{1}^{(I)}}=\frac{\partial n_{1}^{(I)}}{\partial s_{1}} \frac{\partial s_{1}}{\partial p_{1}^{(I)}}=\frac{\partial n_{1}^{(I)}}{\partial s_{1}} \frac{1}{2 t_{1}}\left(1-2 \alpha \frac{\partial n_{2}^{(I)}}{\partial p_{1}^{(I)}}\right), \\
\frac{\partial n_{2}^{(I)}}{\partial p_{1}^{(I)}}=\frac{\partial n_{2}^{(I)}}{\partial s_{2}} \frac{\partial s_{2}}{\partial p_{1}^{(I)}}=\frac{\partial n_{2}^{(I)}}{\partial s_{2}} \frac{1}{2 t_{2}}\left(-2 \beta \frac{\partial n_{1}^{(I)}}{\partial p_{1}^{(I)}}\right),
\end{gathered}
$$

from where it follows that

$$
\begin{gathered}
\frac{\partial n_{1}^{(I)}}{\partial p_{1}^{(I)}}=\frac{1}{2 t_{1}} \frac{\partial n_{1}^{(I)}}{\partial s_{1}}\left(1-\frac{\alpha \beta}{t_{1} t_{2}} \frac{\partial n_{1}^{(I)}}{\partial s_{1}} \frac{\partial n_{2}^{(I)}}{\partial s_{2}}\right)^{-1}, \\
\frac{\partial n_{2}^{(I)}}{\partial p_{1}^{(I)}}=-\frac{\beta}{2 t_{1} t_{2}} \frac{\partial n_{1}^{(I)}}{\partial s_{1}} \frac{\partial n_{2}^{(I)}}{\partial s_{2}}\left(1-\frac{\alpha \beta}{t_{1} t_{2}} \frac{\partial n_{1}^{(I)}}{\partial s_{1}} \frac{\partial n_{2}^{(I)}}{\partial s_{2}}\right)^{-1} .
\end{gathered}
$$

It follows from the symmetry of the problem that in the equilibrium the prices set by the platforms for each group of customers should be equal, although they may differ for different groups, that is, $p_{1}^{(I)}=p_{1}^{(I I)}=p_{1}, p_{2}^{(I)}=p_{2}^{(I I)}=p_{2}$. Furthermore, the size of both groups on both platforms should be equal, that is, $n_{1}^{(I)}=n_{1}^{(I I)}=\frac{1}{2}$ and $n_{2}^{(I)}=n_{2}^{(I I)}=\frac{1}{2}$. Hence $s_{1}=s_{2}=0$ and

$$
\frac{\partial n_{1}^{(I)}}{\partial s_{1}}=\frac{\partial n_{2}^{(I)}}{\partial s_{2}}=-\frac{1}{2} I
$$

It follows that the expression (9) has the form

$$
\frac{\partial H^{(I)}}{\partial p_{1}^{(I)}}=\frac{\partial n_{1}^{(I)}}{\partial p_{1}^{(I)}}\left(p_{1}-g_{1}\right)+\frac{\partial n_{2}^{(I)}}{\partial p_{1}^{(I)}}\left(p_{2}-g_{2}\right)+\frac{1}{2}
$$




$$
=-\frac{1}{8 t_{1} t_{2}} I\left(1-\frac{\alpha \beta}{4 t_{1} t_{2}} I^{2}\right)^{-1}\left(2 t_{2}\left(p_{1}-g_{1}\right)+\beta I\left(p_{2}-g_{2}\right)\right)+\frac{1}{2}=0
$$

Similar reasoning for equation $\frac{\partial H^{(I)}}{\partial p_{2}^{(I)}}=0$, which has the form

$$
\frac{\partial H^{(I)}}{\partial p_{2}^{(I)}}=\frac{\partial n_{1}^{(I)}}{\partial p_{2}^{(I)}}\left(p_{1}^{(I)}-g_{1}\right)+\frac{\partial n_{2}^{(I)}}{\partial p_{2}^{(I)}}\left(p_{2}^{(I)}-g_{2}\right)+n_{2}^{(I)},
$$

leads to the equation

$$
\begin{gathered}
\frac{\partial H^{(I)}}{\partial p_{2}^{(I)}}=\frac{\partial n_{1}^{(I)}}{\partial p_{2}^{(I)}}\left(p_{1}^{(I)}-g_{1}\right)+\frac{\partial n_{2}^{(I)}}{\partial p_{2}^{(I)}}\left(p_{2}^{(I)}-g_{2}\right)+\frac{1}{2} \\
=-\frac{1}{8 t_{1} t_{2}} I\left(1-\frac{\alpha \beta}{4 t_{1} t_{2}} I^{2}\right)^{-1}\left(\alpha I\left(p_{1}-g_{1}\right)+2 t_{1}\left(p_{2}-g_{2}\right)\right)+\frac{1}{2}=0 .
\end{gathered}
$$

From (10) and (11), we find the prices for the groups in the equilibrium

$$
p_{1}=g_{1}+\left(\frac{2}{I} t_{1}-\beta\right), \quad p_{2}=g_{2}+\left(\frac{2}{I} t_{2}-\alpha\right) .
$$

Hence, the following theorem is valid for the case with identical platforms and different groups of agents.

Theorem 2. In the Hotelling model for a two-sided platform market on the plane with identical platforms, competitive service will take place given that

$$
\max \left\{\frac{\alpha}{t_{2}}, \frac{\beta}{t_{1}}\right\} \leq \frac{2}{I} \approx 1.742
$$

and the service prices in the equilibrium in this case will have the form (12).

\section{Different Platforms and Identical Groups of Agents}

Consider the model of a two-sided market with different platforms located in points $(a, 0)$ and $(b, 0)$ of the square $S$, and with identical groups of agents, which have the same parameters of the power of the group size effect and the transport costs. Assume for definiteness that $a \leq b$. In this case, the utility for group 1 agents from visiting the platform $I$ in the point $(a, 0)$ has the form

$$
u_{1}^{(I)}=\alpha \cdot n_{2}^{(I)}-p_{1}^{(I)}-\sqrt{\left(x_{1}-a\right)^{2}+y_{1}^{2}} \cdot t,
$$

while from visiting the platform $I I$ in the point $(b, 0)$ it is

$$
u_{1}^{(I I)}=\alpha \cdot n_{2}^{(I I)}-p_{1}^{(I I)}-\sqrt{\left(x_{1}-b\right)^{2}+y_{1}^{2}} \cdot t .
$$


The boundary between regions of the market for group 1 is determined from the equation $u_{1}^{(I)}=u_{1}^{(I I)}$, which is solved to get that

$$
\frac{\left(x_{1}-\frac{a+b}{2}\right)^{2}}{s_{1}^{2}}-\frac{y_{1}^{2}}{\left(\frac{b-a}{2}\right)^{2}-s_{1}^{2}}=1,
$$

where $s_{1}=\frac{\alpha\left(n_{2}^{(I I)}-n_{2}^{(I)}\right)+p_{1}^{(I)}-p_{1}^{(I I)}}{2 t}$ and $n_{1}^{(I)}+n_{1}^{(I I)}=1$. The number of group 1 agents visiting the platforms $I$ and $I I$, respectively, is found from the formulas

$$
\begin{aligned}
& n_{1}^{(I)}=\frac{1}{2}+\frac{a+b}{4}-\frac{1}{2} \int_{0}^{1} s_{1} \sqrt{1+\frac{y^{2}}{\left(\frac{b-a}{2}\right)^{2}-s_{1}^{2}}} d y, \\
& n_{1}^{(I I)}=\frac{1}{2}-\frac{a+b}{4}+\frac{1}{2} \int_{0}^{1} s_{1} \sqrt{1+\frac{y^{2}}{\left(\frac{b-a}{2}\right)^{2}-s_{1}^{2}}} d y .
\end{aligned}
$$

The utilities of group 2 agents from visiting platforms $I$ and $I I$ are determined similarly, and equal, respectively

$$
\begin{gathered}
u_{2}^{(I)}=\alpha \cdot n_{1}^{(I)}-p_{2}^{(I)}-\sqrt{\left(x_{2}-a\right)^{2}+y_{2}^{2}} \cdot t, \\
u_{2}^{(I I)}=\alpha \cdot n_{1}^{(I I)}-p_{2}^{(I I)}-\sqrt{\left(x_{2}-b\right)^{2}+y_{2}^{2}} \cdot t,
\end{gathered}
$$

and the boundary between market regions for group 2 is found from the equation of the form

$$
\frac{\left(x_{2}-\frac{a+b}{2}\right)^{2}}{s_{2}^{2}}-\frac{y_{2}^{2}}{\left(\frac{b-a}{2}\right)^{2}-s_{2}^{2}}=1,
$$

where $s_{2}=\frac{\alpha\left(n_{1}^{(I I)}-n_{1}^{(I)}\right)+p_{2}^{(I)}-p_{2}^{(I I)}}{2 t}$ and $n_{2}^{(I)}+n_{2}^{(I I)}=1$. The number of group 2 agents visiting the respective platforms is

$$
\begin{aligned}
& n_{2}^{(I)}=\frac{1}{2}+\frac{a+b}{4}-\frac{1}{2} \int_{0}^{1} s_{2} \sqrt{1+\frac{y^{2}}{\left(\frac{b-a}{2}\right)^{2}-s_{2}^{2}}} d y, \\
& n_{2}^{(I I)}=\frac{1}{2}-\frac{a+b}{4}+\frac{1}{2} \int_{0}^{1} s_{2} \sqrt{1+\frac{y^{2}}{\left(\frac{b-a}{2}\right)^{2}-s_{2}^{2}}} d y .
\end{aligned}
$$

Since the groups of agents in this model are identical, the price equilibrium can be found from the first-order optimality condition for any one of the groups of agents (e.g., the first one) in both platforms:

$$
\frac{\partial H^{(I)}}{\partial p_{1}^{(I)}}=0, \quad \frac{\partial H^{(I I)}}{\partial p_{1}^{(I I)}}=0 .
$$


Therefore,

$$
\left\{\begin{array}{l}
\frac{\partial H^{(I)}}{\partial p_{1}^{(I)}}=\frac{\partial n_{1}^{(I)}}{\partial p_{1}^{(I)}}\left(p_{1}^{(I)}-g\right)+n_{1}^{(I)}+\frac{\partial n_{2}^{(I)}}{\partial p_{1}^{(I)}}\left(p_{2}^{(I)}-g\right)=0 \\
\frac{\partial H^{(I I)}}{\partial p_{1}^{(I I)}}=\frac{\partial n_{1}^{(I I)}}{\partial p_{1}^{(I I)}}\left(p_{1}^{(I I)}-g\right)+n_{1}^{(I I)}+\frac{\partial n_{2}^{(I I)}}{\partial p_{1}^{(I I)}}\left(p_{2}^{(I I)}-g\right)=0
\end{array}\right.
$$

The derivatives $\frac{\partial n_{1}^{(I)}}{\partial p_{1}^{(I)}}$ and $\frac{\partial n_{2}^{(I)}}{\partial p_{1}^{(I)}}$ are taken from the Formulas (6) and (7). The expressions for the derivatives $\frac{\partial n_{1}^{(I I)}}{\partial p_{1}^{(I I)}}$ and $\frac{\partial n_{2}^{(I I)}}{\partial p_{1}^{(I I)}}$ are found in a similar way

$$
\begin{gathered}
\frac{\partial n_{1}^{(I I)}}{\partial p_{1}^{(I I)}}=-\frac{1}{2 t} \frac{\partial n_{1}^{(I I)}}{\partial s_{1}}\left(1-\frac{\alpha^{2}}{t^{2}} \frac{\partial n_{1}^{(I I)}}{\partial s_{1}} \frac{\partial n_{2}^{(I I)}}{\partial s_{2}}\right)^{-1}, \\
\frac{\partial n_{2}^{(I I)}}{\partial p_{1}^{(I I)}}=-\frac{\alpha}{2 t^{2}} \frac{\partial n_{1}^{(I I)}}{\partial s_{1}} \frac{\partial n_{2}^{(I I)}}{\partial s_{2}}\left(1-\frac{\alpha^{2}}{t^{2}} \frac{\partial n_{1}^{(I I)}}{\partial s_{1}} \frac{\partial n_{2}^{(I I)}}{\partial s_{2}}\right)^{-1} .
\end{gathered}
$$

It follows from the symmetry of the problem that in the equilibrium the prices in the platforms should be equal for each group of customers, although they may differ for different groups, that is, $p_{1}^{(I)}=p_{2}^{(I)}=p^{(I)}, p_{1}^{(I I)}=p_{2}^{(I I)}=p^{(I I)}$, and the size of the groups in each platform is equal, that is, $n_{1}^{(I)}=n_{2}^{(I)}=n^{(I)}$ and $n_{1}^{(I I)}=n_{2}^{(I I)}=1-n^{(I)}$, wherefore

$$
s=s_{1}=s_{2}=\frac{\alpha-2 \alpha n^{(I)}+p^{(I)}-p^{(I I)}}{2 t},
$$

and

$$
\frac{\partial n_{1}^{(I)}}{\partial s_{1}}=\frac{\partial n_{2}^{(I)}}{\partial s_{2}}=-\frac{\partial n_{1}^{(I I)}}{\partial s_{1}}=-\frac{\partial n_{2}^{(I I)}}{\partial s_{2}}=-\frac{1}{2} \int_{0}^{1}\left(\sqrt{1+\frac{y^{2}}{\left(\frac{b-a}{2}\right)^{2}-s^{2}}}+\frac{s^{2} y^{2}}{\left(\left(\frac{b-a}{2}\right)^{2}-s^{2}\right)^{2} \sqrt{1+\frac{y^{2}}{\left(\frac{b-a}{2}\right)^{2}-s^{2}}}}\right) d y .
$$

Denote the expression (17) as the function $D(s)$. Then, it follows from (6)-(7) and (14)-(15) that

$$
\begin{gathered}
\frac{\partial n_{1}^{(I)}}{\partial p_{1}^{(I)}}=\frac{\partial n_{1}^{(I I)}}{\partial p_{1}^{(I I)}}=\frac{1}{2 t} D(s)\left(1-\frac{\alpha^{2}}{t^{2}} D^{2}(s)\right)^{-1} \\
\frac{\partial n_{2}^{(I)}}{\partial p_{1}^{(I)}}=\frac{\partial n_{2}^{(I)}}{\partial p_{1}^{(I I)}}=-\frac{\alpha}{2 t^{2}} D^{2}(s)\left(1-\frac{\alpha^{2}}{t^{2}} D^{2}(s)\right)^{-1} .
\end{gathered}
$$

The resultant expressions are substituted into system (13):

$$
\left\{\begin{array}{l}
\frac{1}{2 t} D(s)\left(1-\frac{\alpha^{2}}{t^{2}} D^{2}(s)\right)^{-1}\left(p^{(I)}-g\right)-\frac{\alpha}{2 t^{2}} D^{2}(s)\left(1-\frac{\alpha^{2}}{t^{2}} D^{2}(s)\right)^{-1}\left(p^{(I)}-g\right)+n^{(I)}=0 \\
\frac{1}{2 t} D(s)\left(1-\frac{\alpha^{2}}{t^{2}} D^{2}(s)\right)^{-1}\left(p^{(I I)}-g\right)-\frac{\alpha}{2 t^{2}} D^{2}(s)\left(1-\frac{\alpha^{2}}{t^{2}} D^{2}(s)\right)^{-1}\left(p^{(I I)}-g\right)+1-n^{(I)}=0
\end{array}\right.
$$


where

$$
n^{(I)}=\frac{1}{2}+\frac{a+b}{4}-\frac{1}{2} \int_{0}^{1} s \sqrt{1+\frac{y^{2}}{\left(\frac{b-a}{2}\right)^{2}-s^{2}}} d y .
$$

From system (18) we find that

$$
\left\{\begin{array}{l}
t D(s)\left(p^{(I)}-g\right)-\alpha D^{2}(s)\left(p^{(I)}-g\right)+2 n^{(I)}\left(t^{2}-\alpha^{2} D^{2}(s)\right)=0, \\
t D(s)\left(p^{(I I)}-g\right)-\alpha D^{2}(s)\left(p^{(I I)}-g\right)+2\left(1-n^{(I)}\right)\left(t^{2}-\alpha^{2} D^{2}(s)\right)=0,
\end{array}\right.
$$

and subsequently the optimal prices are found using the formulas

$$
\left\{\begin{array}{l}
p^{(I)}=g-2 n^{(I)} \cdot \frac{t+\alpha D(s)}{D(s)} \\
p^{(I I)}=g-2\left(1-n^{(I)}\right) \cdot \frac{t+\alpha D(s)}{D(s)} .
\end{array}\right.
$$

Theorem 3. In the Hotelling model for a two-sided platform market on the plane with identical agents and different platforms, service prices in the equilibrium satisfy Equations (16), (19) and (20).

From Equations (16) and (20), we find that

$$
p^{(I)}-p^{(I I)}=2\left(1-2 n^{(I)}\right) \cdot \frac{t+\alpha D(s)}{D(s)}=2 t s-\alpha+2 \alpha n^{(I)},
$$

wherefore

$$
n^{(I)}=\frac{1}{2}-\frac{t s D(s)}{3 \alpha D(s)+2 t}
$$

Together with (19), this leads to the equation for finding the parameter $s$ in the equilibrium:

$$
\frac{t s D(s)}{3 \alpha D(s)+2 t}=\frac{1}{2} \int_{0}^{1} s \sqrt{1+\frac{y^{2}}{\left(\frac{b-a}{2}\right)^{2}-s^{2}}} d y-\frac{a+b}{4} .
$$

Thus, optimal prices in the pricing game can be found by finding the parameter $s$ from Equation (22), then finding the size of the group of agents in the first platform using Formula (21), and substituting the resultant parameters into Formula (20). The results of the numerical calculations are shown in Table 1.

Table 1. Numerical results for $g=0, \alpha=1, t=1$.

\begin{tabular}{ccccccccc}
\hline $\boldsymbol{a}$ & $\boldsymbol{b}$ & $\mathbf{s}$ & $\boldsymbol{n}_{\mathbf{1}}$ & $\boldsymbol{n}_{\mathbf{2}}$ & $\boldsymbol{p}^{\boldsymbol{I}}$ & $\boldsymbol{p}^{\boldsymbol{I I}}$ & $\boldsymbol{H}^{\boldsymbol{I}}$ & $\boldsymbol{H}^{\boldsymbol{I I}}$ \\
\hline-1 & 1 & 0 & 0.5 & 0.5 & 0.742 & 0.742 & 0.742 & 0.742 \\
-1 & 0.9 & -0.009 & 0.480 & 0.520 & 0.692 & 0.749 & 0.692 & 0.749 \\
-1 & 0.8 & -0.016 & 0.459 & 0.541 & 0.640 & 0.754 & 0.640 & 0.754 \\
-1 & 0.75 & -0.019 & 0.449 & 0.551 & 0.614 & 0.754 & 0.614 & 0.754 \\
-1 & 0.7 & -0.021 & 0.438 & 0.562 & 0.586 & 0.753 & 0.586 & 0.753 \\
-1 & 0.6 & -0.021 & 0.424 & 0.576 & 0.542 & 0.736 & 0.542 & 0.736 \\
\hline
\end{tabular}

The table shows that when the second platform is shifted to the center, $s$ shifts to the left, which reduces the market for platform $I$. This means that customers in the center are getting closer to platform $I I$. 


\section{Optimal Location of Platforms}

Observe the results of numerical calculations in Table 1. When the location of the first platform $I$ is fixed in the position $a=-1$ and the position of the second platform $b$ changes from 1 to 0.6 the second player's payoff $H^{(I I)}$ first grows and then declines. The maximum is reached in the position $b=0.8$. This means that if the position of the first platform is fixed, the second player should put his/her platform in the position $b=0.8$.

If, however, the second player puts his/her platform in that position, the first player may also relocate. Table 2 shows the calculations simulating this situation. Let the players switch roles. The first player is now in the fixed position $a=-0.8$, while the second player's position is variable. It follows from the numerical calculations shown in Table 2 that his/her optimal position in this situation is $b=0.9$. Continuing this sequence of the best responses, we arrive at the equilibrium location of platforms in the market $a=-0.85, b=0.85$.

Table 2. Numerical results for $g=0, \alpha=1, t=1$.

\begin{tabular}{ccccccccc}
\hline $\boldsymbol{a}$ & $\boldsymbol{b}$ & $\mathbf{s}$ & $\boldsymbol{n}_{\mathbf{1}}$ & $\boldsymbol{n}_{\mathbf{2}}$ & $\boldsymbol{p}^{\boldsymbol{I}}$ & $\boldsymbol{p}^{\boldsymbol{I I}}$ & $\boldsymbol{H}^{\boldsymbol{I}}$ & $\boldsymbol{H}^{\boldsymbol{I I}}$ \\
\hline-0.8 & 1 & 0.016 & 0.541 & 0.459 & 0.754 & 0.640 & 0.754 & 0.640 \\
-0.8 & 0.9 & 0.007 & 0.521 & 0.479 & 0.698 & 0.642 & 0.698 & 0.642 \\
-0.8 & 0.8 & 0 & 0.5 & 0.5 & 0.640 & 0.640 & 0.640 & 0.640 \\
-0.8 & 0.7 & -0.005 & 0.478 & 0.522 & 0.579 & 0.632 & 0.579 & 0.632 \\
-0.8 & 0.6 & -0.006 & 0.454 & 0.546 & 0.515 & 0.620 & 0.615 & 0.620 \\
\hline
\end{tabular}

\section{Different Platforms and Different Groups of Agents}

Consider the case where different platforms serve different groups of agents, which are described by the parameters $\alpha \neq \beta$ and $t_{1} \neq t_{2}$. In this case, the payoffs of both platforms have the form

$$
\left\{\begin{array}{l}
H^{(I)}\left(p_{1}^{(I)}, p_{2}^{(I)}\right)=n_{1}^{(I)}\left(p_{1}^{(I)}-g_{1}\right)+n_{2}^{(I)}\left(p_{2}^{(I)}-g_{2}\right), \\
H^{(I I)}\left(p_{1}^{(I I)}, p_{2}^{(I I)}\right)=\left(1-n_{1}^{(I)}\right)\left(p_{1}^{(I I)}-g_{1}\right)+\left(1-n_{2}^{(I)}\right)\left(p_{2}^{(I)}-g_{2}\right) .
\end{array}\right.
$$

The price equilibrium for the platforms is found from the first-order optimality condition

$$
\frac{\partial H^{(I)}}{\partial p_{i}^{(I)}}=0, \quad \frac{\partial H^{(I I)}}{\partial p_{i}^{(I I)}}=0, \quad i=1,2
$$

Therefore,

$$
\left\{\begin{array}{l}
\frac{\partial H^{(I)}}{\partial p_{1}^{(I)}}=\frac{\partial n_{1}^{(I)}}{\partial p_{1}^{(I)}}\left(p_{1}^{(I)}-g_{1}\right)+n_{1}^{(I)}+\frac{\partial n_{2}^{(I)}}{\partial p_{1}^{(I)}}\left(p_{2}^{(I)}-g_{2}\right)=0, \\
\frac{\partial H^{(I)}}{\partial p_{2}^{(I)}}=\frac{\partial n_{1}^{(I)}}{\partial p_{2}^{(I)}}\left(p_{1}^{(I)}-g_{1}\right)+n_{2}^{(I)}+\frac{\partial n_{2}^{(I)}}{\partial p_{2}^{(I)}}\left(p_{2}^{(I)}-g_{2}\right)=0, \\
\frac{\partial H^{(I I)}}{\partial p_{1}^{(I I)}}=-\frac{\partial n_{1}^{(I)}}{\partial p_{1}^{(I I)}}\left(p_{1}^{(I I)}-g_{1}\right)+1-n_{1}^{(I I)}-\frac{\partial n_{2}^{(I)}}{\partial p_{1}^{(I I)}}\left(p_{2}^{(I I)}-g_{2}\right)=0, \\
\frac{\partial H^{(I I)}}{\partial p_{2}^{(I I)}}=-\frac{\partial n_{1}^{(I)}}{\partial p_{2}^{(I I)}}\left(p_{1}^{(I I)}-g_{1}\right)+1-n_{2}^{(I I)}-\frac{\partial n_{2}^{(I)}}{\partial p_{2}^{(I I)}}\left(p_{2}^{(I I)}-g_{2}\right)=0,
\end{array}\right.
$$

where the respective derivatives satisfy the following equations:

$$
\frac{\partial n_{1}^{(I)}}{\partial p_{1}^{(I)}}=-\frac{\partial n_{1}^{(I)}}{\partial p_{1}^{(I I)}}=\frac{1}{2 t_{1}} \frac{\partial n_{1}^{(I)}}{\partial s_{1}}\left(1-\frac{\alpha \beta}{t_{1} t_{2}} \frac{\partial n_{1}^{(I)}}{\partial s_{1}} \frac{\partial n_{2}^{(I)}}{\partial s_{2}}\right)^{-1}
$$




$$
\begin{gathered}
\frac{\partial n_{2}^{(I)}}{\partial p_{1}^{(I)}}=\frac{\partial n_{2}^{(I)}}{\partial p_{1}^{(I)}}=-\frac{\beta}{2 t_{1} t_{2}} \frac{\partial n_{1}^{(I)}}{\partial s_{1}} \frac{\partial n_{2}^{(I)}}{\partial s_{2}}\left(1-\frac{\alpha \beta}{t_{1} t_{2}} \frac{\partial n_{1}^{(I)}}{\partial s_{1}} \frac{\partial n_{2}^{(I)}}{\partial s_{2}}\right)^{-1}, \\
\frac{\partial n_{1}^{(I)}}{\partial p_{2}^{(I)}}=-\frac{\partial n_{1}^{(I)}}{\partial p_{2}^{(I I)}}=-\frac{\alpha}{2 t_{1} t_{2}} \frac{\partial n_{1}^{(I)}}{\partial s_{1}} \frac{\partial n_{2}^{(I)}}{\partial s_{2}}\left(1-\frac{\alpha \beta}{t_{1} t_{2}} \frac{\partial n_{1}^{(I)}}{\partial s_{1}} \frac{\partial n_{2}^{(I)}}{\partial s_{2}}\right)^{-1}, \\
\frac{\partial n_{2}^{(I)}}{\partial p_{2}^{(I)}}=-\frac{\partial n_{2}^{(I)}}{\partial p_{2}^{(I I)}}=\frac{1}{2 t_{2}} \frac{\partial n_{2}^{(I)}}{\partial s_{2}}\left(1-\frac{\alpha \beta}{t_{1} t_{2}} \frac{\partial n_{1}^{(I)}}{\partial s_{1}} \frac{\partial n_{2}^{(I)}}{\partial s_{2}}\right)^{-1} .
\end{gathered}
$$

The following notations are introduced:

$$
\begin{aligned}
& D\left(s_{1}\right)=\frac{\partial n_{1}^{(I)}}{\partial s_{1}}=-\frac{1}{2} \int_{0}^{1}\left(\sqrt{1+\frac{y^{2}}{\left(\frac{b-a}{2}\right)^{2}-s_{1}^{2}}}+\frac{s_{1}^{2} y^{2}}{\left(\left(\frac{b-a}{2}\right)^{2}-s_{1}^{2}\right)^{2} \sqrt{1+\frac{y^{2}}{\left(\frac{b-a}{2}\right)^{2}-s_{1}^{2}}}}\right) d y, \\
& D\left(s_{2}\right)=\frac{\partial n_{2}^{(I)}}{\partial s_{2}}=-\frac{1}{2} \int_{0}^{1}\left(\sqrt{1+\frac{y^{2}}{\left(\frac{b-a}{2}\right)^{2}-s_{2}^{2}}}+\frac{s_{2}^{2} y^{2}}{\left(\left(\frac{b-a}{2}\right)^{2}-s_{2}^{2}\right)^{2} \sqrt{1+\frac{y^{2}}{\left(\frac{b-a}{2}\right)^{2}-s_{2}^{2}}}}\right) d y .
\end{aligned}
$$

It follows from Formulas (24)-(29) that

$$
\begin{aligned}
& \frac{\partial n_{1}^{(I)}}{\partial p_{1}^{(I)}}=\frac{1}{2 t_{1}} D\left(s_{1}\right)\left(1-\frac{\alpha \beta}{t_{1} t_{2}} D\left(s_{1}\right) D\left(s_{2}\right)\right)^{-1}, \quad \frac{\partial n_{2}^{(I)}}{\partial p_{1}^{(I)}}=-\frac{\beta}{2 t_{1} t_{2}} D\left(s_{1}\right) D\left(s_{2}\right)\left(1-\frac{\alpha \beta}{t_{1} t_{2}} D\left(s_{1}\right) D\left(s_{2}\right)\right)^{-1}, \\
& \frac{\partial n_{1}^{(I)}}{\partial p_{2}^{(I)}}=-\frac{\alpha}{2 t_{1} t_{2}} D\left(s_{1}\right) D\left(s_{2}\right)\left(1-\frac{\alpha \beta}{t_{1} t_{2}} D\left(s_{1}\right) D\left(s_{2}\right)\right)^{-1}, \quad \frac{\partial n_{2}^{(I)}}{\partial p_{2}^{(I)}}=\frac{1}{2 t_{2}} D\left(s_{2}\right)\left(1-\frac{\alpha \beta}{t_{1} t_{2}} D\left(s_{1}\right) D\left(s_{2}\right)\right)^{-1},
\end{aligned}
$$

and the system of Equation (23) is transformed into the following:

$$
\left\{\begin{array}{l}
t_{2} D\left(s_{1}\right)\left(p_{1}^{(I)}-g_{1}\right)+2 t_{1} t_{2} n_{1}^{(I)}\left(1-\frac{\alpha \beta}{t_{1} t_{2}} D\left(s_{1}\right) D\left(s_{2}\right)\right)-\beta D\left(s_{1}\right) D\left(s_{2}\right)\left(p_{2}^{(I)}-g_{2}\right)=0, \\
-\alpha D\left(s_{1}\right) D\left(s_{2}\right)\left(p_{1}^{(I)}-g_{1}\right)+2 t_{1} t_{2} n_{2}^{(I)}\left(1-\frac{\alpha \beta}{t_{1} t_{2}} D\left(s_{1}\right) D\left(s_{2}\right)\right)+t_{1} D\left(s_{2}\right)\left(p_{2}^{(I)}-g_{2}\right)=0, \\
t_{2} D\left(s_{1}\right)\left(p_{1}^{(I I)}-g_{1}\right)+2 t_{1} t_{2}\left(1-n_{1}^{(I)}\right)\left(1-\frac{\alpha \beta}{t_{1} t_{2}} D\left(s_{1}\right) D\left(s_{2}\right)\right)-\beta D\left(s_{1}\right) D\left(s_{2}\right)\left(p_{2}^{(I I)}-g_{2}\right)=0, \\
-\alpha D\left(s_{1}\right) D\left(s_{2}\right)\left(p_{1}^{(I I)}-g_{1}\right)+2 t_{1} t_{2}\left(1-n_{2}^{(I)}\right)\left(1-\frac{\alpha \beta}{t_{1} t_{2}} D\left(s_{1}\right) D\left(s_{2}\right)\right)+t_{1} D\left(s_{2}\right)\left(p_{2}^{(I I)}-g_{2}\right)=0,
\end{array}\right.
$$

where

$$
\begin{aligned}
& s_{1}=\frac{\alpha-2 \alpha n_{2}^{(I)}+p_{1}^{(I)}-p_{1}^{(I I)}}{2 t_{1}}, \\
& s_{2}=\frac{\beta-2 \beta n_{1}^{(I)}+p_{2}^{(I)}-p_{2}^{(I I)}}{2 t_{2}},
\end{aligned}
$$




$$
\begin{aligned}
& n_{1}^{(I)}=\frac{1}{2}+\frac{a+b}{4}-\frac{1}{2} \int_{0}^{1} s_{1} \sqrt{1+\frac{y^{2}}{\left(\frac{b-a}{2}\right)^{2}-s_{1}^{2}}} d y, \\
& n_{2}^{(I)}=\frac{1}{2}+\frac{a+b}{4}-\frac{1}{2} \int_{0}^{1} s_{2} \sqrt{1+\frac{y^{2}}{\left(\frac{b-a}{2}\right)^{2}-s_{2}^{2}}} d y .
\end{aligned}
$$

The system of Equation (30) yields formulas for finding the optimal prices in the equilibrium:

$$
\left\{\begin{array}{l}
p_{1}^{(I)}=g_{1}-\frac{2\left(\beta D\left(s_{1}\right) n_{2}^{(I)}+t_{1} n_{1}^{(I)}\right)}{D\left(s_{1}\right)} \\
p_{2}^{(I)}=g_{2}-\frac{2\left(\alpha D\left(s_{2}\right) n_{1}^{I}+t_{2} n_{2}^{I}\right)}{D\left(s_{2}\right)} \\
p_{1}^{(I I)}=g_{1}-\frac{2\left(\beta D\left(s_{1}\right)\left(1-n_{2}^{I}\right)+t_{1}\left(1-n_{1}^{I}\right)\right)}{D\left(s_{1}\right)} \\
p_{2}^{(I I)}=g_{2}-\frac{2\left(\alpha D\left(s_{2}\right)\left(1-n_{1}^{I}\right)+t_{2}\left(1-n_{2}^{I}\right)\right)}{D\left(s_{2}\right)}
\end{array}\right.
$$

Thus, the following theorem is valid for the case of different platforms and different agents.

Theorem 4. In the Hotelling model for a two-sided platform market on the plane with different agents and different platforms, service prices in the equilibrium satisfy Equations (30)-(35).

From Equations (31), (32) and (35), we have that

$$
\begin{aligned}
& p_{1}^{(I)}-p_{1}^{(I I)}=\frac{2\left(\beta D\left(s_{1}\right)\left(1-2 n_{2}^{I}\right)+t_{1}\left(1-2 n_{1}^{I}\right)\right)}{D\left(s_{1}\right)}=2 s_{1} t_{1}-\alpha+2 \alpha n_{2}^{(I)}, \\
& p_{2}^{(I)}-p_{2}^{(I I)}=\frac{2\left(\alpha D\left(s_{2}\right)\left(1-2 n_{1}^{I}\right)+t_{2}\left(1-2 n_{2}^{I}\right)\right)}{D\left(s_{2}\right)}=2 s_{2} t_{2}-\beta+2 \beta n_{2}^{(I)},
\end{aligned}
$$

from where the number of agents in the first platform is determined using the formulas

$$
\begin{aligned}
& n_{1}^{(I)}=\frac{1}{2}-\frac{t_{2} D\left(s_{1}\right)\left(s_{2} D\left(s_{2}\right)(2 \beta+\alpha)-2 s_{1} t_{1}\right)}{D\left(s_{1}\right) D\left(s_{2}\right)(2 \beta+\alpha)(2 \alpha+\beta)-4 t_{1} t_{2}} \\
& n_{2}^{I}=\frac{1}{2}-\frac{t_{1} D\left(s_{2}\right)\left(s_{1} D\left(s_{1}\right)(2 \alpha+\beta)-2 s_{2} t_{2}\right)}{D\left(s_{1}\right) D\left(s_{2}\right)(2 \beta+\alpha)(2 \alpha+\beta)-4 t_{1} t_{2}} .
\end{aligned}
$$

The Equations (36) and (37) together with (33) and (34) lead to the system of equations for finding the parameters $s_{1}$ and $s_{2}$ in the equilibrium:

$$
\left\{\begin{array}{l}
\frac{t_{2} D\left(s_{1}\right)\left(s_{2} D\left(s_{2}\right)(2 \beta+\alpha)-2 s_{1} t_{1}\right)}{D\left(s_{1}\right) D\left(s_{2}\right)(2 \beta+\alpha)(2 \alpha+\beta)-4 t_{1} t_{2}}=\frac{a+b}{4}-\frac{1}{2} \int_{0}^{1} s_{1} \sqrt{1+\frac{y^{2}}{\left(\frac{b-a}{2}\right)^{2}-s_{1}^{2}}} d y, \\
\frac{t_{1} D\left(s_{2}\right)\left(s_{1} D\left(s_{1}\right)(2 \alpha+\beta)-2 s_{2} t_{2}\right)}{D\left(s_{1}\right) D\left(s_{2}\right)(2 \beta+\alpha)(2 \alpha+\beta)-4 t_{1} t_{2}}=\frac{a+b}{4}-\frac{1}{2} \int_{0}^{1} s_{2} \sqrt{1+\frac{y^{2}}{\left(\frac{b-a}{2}\right)^{2}-s_{2}^{2}}} d y .
\end{array}\right.
$$

Thus, the values of the parameters $s_{1}$ and $s_{2}$ are found from the system of Equation (38), then the sizes of the first and second groups on the first platform are found using the Formulas (36) and (37), 
and the optimal prices the platforms charge different groups are determined by the Formula (35). The results of numerical calculations are shown in Table 3 for different values of the parameters $\alpha$ and $\beta$.

Table 3. Numerical results for $\alpha=1$ and $\beta=0.7$ when $t_{1}=t_{2}=1$ and $g_{1}=g_{2}=0$.

\begin{tabular}{cccccccccccccc}
\hline $\boldsymbol{a}$ & $\boldsymbol{b}$ & $\boldsymbol{s}_{\mathbf{1}}$ & $\boldsymbol{s}_{\mathbf{2}}$ & $\boldsymbol{n}_{\mathbf{1}}^{\boldsymbol{I}}$ & $\boldsymbol{n}_{\mathbf{1}}^{\boldsymbol{I}}$ & $\boldsymbol{n}_{\mathbf{2}}^{\boldsymbol{I}}$ & $\boldsymbol{n}_{\mathbf{2}}^{\boldsymbol{I I}}$ & $\boldsymbol{p}_{\mathbf{1}}^{\boldsymbol{I}}$ & $\boldsymbol{p}_{\mathbf{1}}^{\boldsymbol{I I}}$ & $\boldsymbol{p}_{\mathbf{2}}^{\boldsymbol{I}}$ & $\boldsymbol{p}_{\mathbf{2}}^{\boldsymbol{I I}}$ & $\boldsymbol{H}^{\boldsymbol{I}}$ & $\boldsymbol{H}^{\boldsymbol{I I}}$ \\
\hline-1 & 1 & 0 & 0 & 0.5 & 0.5 & 0.5 & 0.5 & 1.04 & 1.04 & 0.742 & 0.742 & 0.892 & 0.892 \\
-1 & 0.9 & 0.057 & -0.064 & 0.508 & 0.492 & 0.488 & 0.512 & 1.064 & 0.973 & 0.660 & 0.776 & 0.863 & 0.875 \\
-1 & 0.8 & 0.113 & -0.126 & 0.518 & 0.482 & 0.476 & 0.524 & 1.076 & 0.897 & 0.571 & 0.797 & 0.828 & 0.851 \\
-1 & 0.7 & 0.167 & -0.182 & 0.525 & 0.475 & 0.465 & 0.535 & 1.073 & 0.809 & 0.471 & 0.800 & 0.782 & 0.812 \\
-1 & 0.6 & 0.217 & -0.235 & 0.534 & 0.466 & 0.454 & 0.546 & 1.051 & 0.707 & 0.356 & 0.779 & 0.723 & 0.754 \\
-1 & 0.5 & 0.265 & -0.289 & 0.544 & 0.456 & 0.444 & 0.556 & 1.000 & 0.583 & 0.220 & 0.725 & 0.642 & 0.669 \\
\hline
\end{tabular}

\section{Conclusions}

This paper investigated the structure of prices in the equilibrium in a two-sided market on the plane. Service is provided in two platforms for heterogeneous agents and endogenous demand. Agents are uniformly distributed in a square. The target functions are the platform's maximum profit. The exact expressions were found for the prices which depend on the cost structure and network externalities. Analytical expressions were derived for equilibrium prices. The numerical experiments for finding the equilibrium prices in the market for different parameters of the problem and different locations of platforms in the market were described. The study was concerned with the case where agents of both groups were uniformly distributed over the market. We plan to study this problem in application to different distributions of agents in space.

The paper demonstrated the validity of the problem of the optimal platform location in the market. In the future, the optimal platform location problem will be considered in more detail for various distributions of agents over the market and for a greater number of competing platforms.

Author Contributions: Formal analysis, V.M. and E.K.; Investigation, E.K.; Methodology, V.M. All authors have read and agreed to the published version of the manuscript.

Funding: This research was funded by the Shandong Province “Double-Hundred Talent Plan" (No. WST2017009).

Conflicts of Interest: The authors declare no conflict of interest.

\section{References}

1. Armstrong, M. Competition in two-sided markets. RAND J. Econ. 2006, 37, 668-691. [CrossRef]

2. Hotelling, H. Stability in competition. Econ. J. 1929, 39, 41-57. [CrossRef]

3. Baye, M.R.; Morgan, J. Information Gatekeepers on the Internet and the Competitiveness of Homogenous Product Markets. Am. Econ. Rev. 2001, 91, 454-474. [CrossRef]

4. Parker, G.; Van Alstyne, M. Information Complements, Substitutes, and Strategic Product Design; Mimeo, Tulane University and University of Michigan: New Orleans, LA, USA, 2000.

5. Rochet, J.C.; Tirole, J. Platform Competition in Two-Sided Markets. J. Eur. Econ. Assoc. 2003, 1, $990-1029$. [CrossRef]

6. Rochet, J.C.; Tirole, J. Two-Sided Markets: A Progress Report. RAND J. Econ. 2006, 37, 645-667. [CrossRef]

7. Schmalensee, R. Payment Systems and Interchange Fees. J. Ind. Econ. 2002, 50, 103-122. [CrossRef]

8. Rochet, J.C.; Tirole, J. Cooperation among Competitors: The Economics of Payment Card Associations. Rand J. Econ. 2002, 33, 1-22.

9. Armstrong, M.; Wright, J. Two-Sided Markets, Competitive Bottlenecks and Exclusive Contracts. Econ. Theory 2007, 32, 353-380. [CrossRef]

10. Caillaud, B.; Jullien, B. Chicken and Egg: Competition Among Intermediation Service Providers. RAND J. Econ. 2003, 34, 309-328. [CrossRef]

11. Feng, Z.; Liu, T.; Mazalov, V.V.; Zheng, J. Pricing of platforms in two-sided markets with heterogeneous agents and limited market size. Autom. Remote Control 2019, 80, 1347-1357. [CrossRef] 
12. Bester, H.; De Palma, A.; Leininger, W.; Thomas, J.; Von Thadden, E.L. A non-cooperative analysis of Hotelling's location game. Games Econ. Behav. 1996, 12, 165-186. [CrossRef]

13. Eiselt, H.A.; Marianov, V. Foundations of Location Analysis; International Series in Operations Research \& Management Science; Springer Science \& Business Media: Berlin, Germany, 2011; Volume 115, 510p.

14. Mallozzi, L. Noncooperative facility location games. Oper. Res. Lett. 2007, 35, 151-154.

15. Mazalov, V.V.; Sakaguchi, M. Location game on the plane. Int. Game Theory Rev. 2003, 5, 13-25.

16. Li, M.; Lien, J.W.; Zheng, J. First Mover Advantage versus Price Advantage-The Role of Network Effect in the Competition between Proprietary Software (PS) and Opensource Software (OSS); Tsinghua University: Beijing, China, 2017.

17. Li, M.; Lien, J.W.; Zheng, J. Timing in Bricks Versus Clicks: Real and Virtual Competition with Sequential Entrants; Tsinghua University: Beijing, China, 2018.

18. Li, M.; Zheng, J. Open Source Software Movement: A Challenging Opportunity for the Development of China's Software Industry. J. Electron. Sci. Technol. China 2004, 2, 47-52.

19. Lien, J.W.; Mazalov, V.V.; Melnik, A.V.; Zheng, J. Wardrop Equilibrium for Networks with the BPR Latency Function. In Lecture Notes in Computer Science: Discrete Optimization and Operations Research, Proceedings of the 9th International Conference, DOOR 2016, Vladivostok, Russia, 19-23 September 2016; Springer: Cham, Switzerland, 2016; Volume 9869, pp. 37-49.

20. Mazalov, V.V.; Chirkova, Y.V.; Zheng, J.; Lien, J.W. A Game-Theoretic Model of Virtual Operators Competition in a Two-Sided Telecommunication Market. Autom. Remote Control 2018, 79, 737-756.

21. Mazalov, V.V.; Melnik, A.V. Equilibrium Prices and Flows in the Passenger Traffic Problem. Int. Game Theory Rev. 2016, 18, 1-19. [CrossRef]

(C) 2020 by the authors. Licensee MDPI, Basel, Switzerland. This article is an open access article distributed under the terms and conditions of the Creative Commons Attribution (CC BY) license (http:/ / creativecommons.org/licenses/by/4.0/). 